\title{
Escala de autoestima coletiva: Evidências de validade fatorial e consistência interna
}

\author{
Valdiney Veloso Gouveia \\ Maria Gabriela Costa Ribeiro \\ Alessandro Teixeira Rezende \\ Thiago Medeiros Cavalcanti \\ Heloísa Bárbara Cunha Moizeís \\ Alex Sandro de Moura Grangeiro
}

\section{RESUMO}

O objetivo deste artigo foi adaptar para o contexto brasileiro a Escala de Autoestima Coletiva (EAC), reunindo evidências de sua validade fatorial e consistência interna. Realizaram-se dois estudos. No Estudo 1 participaram 497 estudantes universitários, a maioria do sexo masculino $(51,5 \%)$ com idade média de 21 anos. Estes responderam a EAC e perguntas sociodemográficas. Os resultados apoiaram o modelo original com quatro fatores oblíquos de autoestima, que apresentaram alfas de Cronbach variando de 0,53 (pública) a 0,82 (privada). 0 Estudo 2 reuniu 391 pessoas da população geral, que responderam a EAC e perguntas sociodemográficas, os quais eram predominantemente do sexo masculino $(60,9 \%)$ e com idade média de 23 anos. Os resultados corroboraram a adequação da estrutura fatorial anteriormente indicada, cujos fatores apresentaram alfas de Cronbach entre 0,59 (pública) a 0,85 (privada). Concluindo, embora possa ser considerada uma pontuação total desta medida de autoestima coletiva, que apresentou consistência interna satisfatória nos dois estudos ( $\mathrm{a}=$ 0,80 e 0,84 , respectivamente), é possível considerar igualmente seus fatores individuais, conforme propuseram seus autores.

Palavras-chave: autoestima; coletiva; validade; precisão; escala.

\section{ABSTRACT \\ Collective Self-Esteem Scale: Evidence of Factorial Validity and Reliability}

This study aimed at adapting the Collective Self-Esteem Scale (CSES) to the Brazilian context, gathering evidence of its factorial validity and reliability. Two studies were carried out. In Study 1, 497 university students participated, most of them males (51.5\%), with a mean age of 21 years. They answered the CSES and sociodemographic questions. Results supported the original model with four oblique self-esteem factors, which presented Cronbach's alphas ranging from 0.53 (public) to 0.82 (private). In Study 2 participants were 391 people from the general population, who answered the CSES and sociodemographic questions, being predominantly male (60.9\%), with a mean age of 23 years. Results corroborated the adequacy of the factorial structure previously indicated, whose factors presented Cronbach's alphas between 0.59 (public) and 0.85 (private). In conclusion, although it can be considered a total score of this measure of collective self-esteem, which presented satisfactory reliability in the two studies ( $a=0.80$ and 0.84 , respectively), it is possible to also consider its specific factors, as proposed by authors of this scale.

Keywords: self-esteem; collective; validity; reliability; scale.

A autoestima pode ser compreendida como um elemento avaliativo do autoconceito, retratando aspectos comportamentais, cognitivos, valorativos e afetivos característicos de determinado indivíduo. No caso, esta avaliação, que é feita pela própria pessoa, pode ser orientada

\author{
Sobre os Autores \\ V. V. G. \\ orcid.org/0000-0003-2107-5848 \\ Universidade Federal da Paraíba \\ (UFPB) - João Pessoa, PB \\ vvgouveia@gmail.com
}

M. G. C. R.

orcid.org/0000-0001-6920-9070 Universidade Federal da Paraíba (UFPB) - João Pessoa, PB mariagabicr@gmail.com

A. T. R.

orcid.org/0000-0002-5381-2155 Universidade Federal da Paraíba (UFPB) - João Pessoa, PB als_tx29@hotmail.com

T. M. C.

orcid.org/0000-0003-4554-7539

Universidade Federal da Paraíba (UFPB) - João Pessoa, PB

thiagomcavalcantii@gmail.com

H. B. C. M.

orcid.org/0000-0003-0477-8410

Universidade Federal da Paraíba

(UFPB) - João Pessoa, PB

heloisabarbara96@gmail.com

A. S. M. G.

orcid.org/0000-0003-0477-8410

Faculdade Luciano Feijão -

Sobral, CE

alexsmgrangeiro@gmail.com

\section{Direitos Autorais}

Este é um artigo de acesso aberto e pode ser reproduzido livremente, distribuído,

transmitido ou modificado, por qualquer pessoa desde que usado sem fins comerciais. 0 trabalho é disponibilizado sob a licença Creative Commons CCBY-NC.

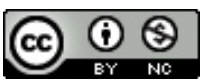




\section{INTERACÃO EM PSICOLOGIA}

Valdiney Veloso Gouveia, Maria Gabriela Costa Ribeiro, Alessandro Teixeira Rezende, Thiago Medeiros Cavalcanti, Heloisa Bárbara Cunha Moizeís e Alex Sandro de Moura Grangeiro por autoaprovação e/ou depreciação; a primeira constitui um aspecto positivo que o indivíduo nutre de si mesmo, enquanto que a segunda parte de uma perspectiva mais negativa que a pessoa forma a seu respeito (Hutz \& Zanon, 2011; Kernis, 2005). Esta dimensão psicológica tem se revelado importante, observando-se, por exemplo, que a autoestima positiva costuma estar correlacionada diretamente com a satisfação com a vida (Diener \& Diener, 2009), fazendo-o inversamente com a depressão e a ansiedade (Sowislo \& Orth, 2013).

Du, Ronnel e King (2012) sugerem que os indivíduos podem avaliar sua autoestima por meio de três elementos, a saber: autoestima pessoal (percepção do indivíduo em relação aos próprios atributos), autoestima coletiva (concepção da pessoa em relação ao seu valor dentro do grupo social) e autoestima relacional (julgamentos positivos a partir das relações significativas do indivíduo, como, por exemplo, com sua família e seus amigos). Esse elemento se diferencia dos demais em razão da importância dada aos pares bem como na especificação dessas relações, não se enquadrando em um grupo social ampliado. Entretanto, a sua avaliação possui limitação, uma vez que se concentra em contextos relacionais mais específicos em que a família, os amigos e os professores, por exemplo, são mais inclinados a serem considerados como um grupo social primário (Andersen \& Cole, 1990).

Desse modo, pode-se enquadrar esse tipo na dimensão de autoestima coletiva, que, por sua vez, é construída a partir de relações que se estabelecem com os grupos sociais. Ressalta-se que embora a autoestima pessoal e coletiva sejam distintas uma da outra, elas estão relacionadas; ambas se sustentam no sentido de garantir o valor do indivíduo ou grupo, revestindo-se da mesma função de proteção contra uma ameaça. A autoestima coletiva protege mais o valor da pessoa de uma ameaça à identidade social (coletiva) do que pessoal; diferentemente, a autoestima pessoal protege o autovalor da pessoa mais de uma ameaça à sua identidade pessoal (Bazińska, 2015; Hassan, Rousseau, \& Moreau, 2013).

A ênfase neste estudo é com a autoestima coletiva, que é concebida como indistinta da relacional, considerando que para o funcionamento do homem como um ser social é vital sua pertença a diferentes grupos sociais, incluindo os primários (e.g., família, amigos íntimos). A propósito, há que se dizer que a pertença a um grupo proporciona sentimento de proteção e fortalecimento de identidade grupal (Bazińska, 2015; Simsek, 2013). Além disso, possibilita uma imagem positiva ou negativa acerca de si mesmo, que pode resultar em uma avaliação favorável ou desfavorável dos grupos nos quais a pessoa está inserida, não se restringindo apenas ao âmbito individual (Tajfel, 1981). Desse modo, os sentimentos de autorrespeito e autovalorização, que surgem por meio da inserção do indivíduo em um grupo social, retratam sua autoestima coletiva (Crocker, Luhtanen, Blaine, \& Broadnax, 1994).

Luhtanen e Crocker (1992) advogam pela legitimidade da autoestima coletiva a partir das diferenças individuais que existem na identidade social. Por meio de relações e comparações intergrupais, a autoestima coletiva emerge quando são confrontadas as imagens dos grupos sociais aos quais os indivíduos pertencem. Nesse caso, na medida em que a imagem do grupo ao qual a pessoa pertence é positiva, o nível de autoestima coletiva se apresenta mais alto; em contrapartida, quando a comparação com seu grupo resulta em uma imagem desvantajosa, a autoestima coletiva tende a ser menor (Suit, 2012).

Tendo em mente esta concepção da autoestima, Luhtanen e Crocker (1992) desenvolveram a Collective Self-Esteem Scale, que avalia a autoestima coletiva em quatro domínios: filiação, privada, pública e identidade. A partir de sua construção, alguns estudos têm procurado adaptar e reunir evidências psicométricas desta medida, tentando identificar os correlatos da autoestima coletiva (para uma revisão, ver Bazińska, 2015). Entretanto, apesar de estudos no Brasil terem usado esta medida, contando com sua tradução (Melo, 2014; Paiva \& Romero, 2004; Souza \& Ferreira, 2005; Suit, 2012), não se tem informação sobre qualquer tentativa de conhecer seus parâmetros psicométricos, o que motiva o presente estudo; antes de apresentá-lo, entretanto, é importante ter em conta a elaboração e os parâmetros psicométricos desta medida.

\section{ESCALA DE AUTOESTIMA COLETIVA}

A maioria das medidas de autoestima disponível na literatura se concentra na avaliação dos indivíduos apenas nas dimensões de identidade privada, pessoal ou interpessoal, inexistindo até então instrumentos que avaliem o domínio coletivo da identidade e de pertença grupal. Na tentativa de preencher essa lacuna, Luhtanen e Crocker (1992) desenvolveram a Escala de Autoestima Coletiva, pretendendo medir a avaliação que os indivíduos fazem da sua autoestima em relação à cultura e ao grupo social nos quais estão inseridos.

Nesta direção, estes autores pretenderam incluir quatro dimensões capazes de avaliar os aspectos coletivos da autoestima, como seguem: (1) filiação: esta dimensão mensura o sentimento de filiação, refletindo o julgamento do quanto dignos os indivíduos se sentem enquanto membros de seus grupos sociais; (2) privada: nesta dimensão procura-se avaliar julgamentos pessoais de como os indivíduos se sentem enquanto pertencentes a um grupo social; (3) pública: esta terceira dimensão procura cobrir a ideia de como as pessoas 


\section{INTERACÃO EM PSICOLOGIA}

Valdiney Veloso Gouveia, Maria Gabriela Costa Ribeiro, Alessandro Teixeira Rezende, Thiago Medeiros Cavalcanti, Heloisa Bárbara Cunha Moizeís e Alex Sandro de Moura Grangeiro avaliam as demais do grupo social do qual fazem parte; e, por último, (4) identidade: esta dimensão cobre a importância da pertença grupal na formação do autoconceito no indivíduo.

Inicialmente, Luhtanen e Crocker (1992) consideraram diversos pressupostos teóricos para desenvolver sua medida, elaborando dez itens para cada uma das quatro dimensões da autoestima coletiva, além de outros três destinados a avaliar a importância de ser um bom membro do grupo, o sentimento individual acerca de seus próprios grupos e o sentimento que as outras pessoas em geral têm em relação a tais grupos. Portanto, a versão preliminar desta medida reuniu 43 itens, sendo aplicada a uma amostra de 82 indivíduos. Os dados correspondentes foram submetidos a uma análise de componentes principais (rotação varimax), indicando que os quatro fatores esperados explicavam $55,2 \%$ da variância total. Posteriormente, visando diminuir o tamanho desta escala, seus autores selecionaram quatro itens de cada subescala, resultando na medida final composta por 16 itens. 0 critério para a exclusão dos itens teve como base os valores da carga fatorial, tendo assumido valores acima de 0,70 (com exceção de dois itens com cargas de 0,65 e 0,62).

A versão final, constituída por 16 itens, considerando o mesmo grupo de participantes $(n=82)$, foi novamente submetida a uma análise de componentes principais (rotação varimax), mostrando que os quatro fatores explicaram $72,3 \%$ da variância total; seus itens tiveram cargas fatoriais entre $0,58 \mathrm{e}$ 0,88 . No que concerne à consistência interna das subescalas, os alfas de Cronbach variaram entre 0,83 e 0,88, tendo correlação média inter-itens superior a 0,50. Estas subescalas mostraram correlações entre elas, que variaram entre 0,21 (Público e Identidade, $p<0,05$ ) e 0,51 (Filiação e Privada, $p<$ $0,01)$. As pontuações desta medida se correlacionaram com a Escala de Autoestima de Rosenberg $(r=0,34, p<0,01)$, indicando que as autoestimas individual e coletiva são moderadamente correlacionadas. Por outro lado, corroborando a concepção de que a subescala mais individualista da medida de autoestima coletiva se correlacionaria com a autoestima pessoal (Rosenberg), o fator filiação foi o que teve correlação mais forte $(r=0,47, p<0,001)$.

Considerando o tamanho reduzido da amostra inicial, Luhtanen e Crocker (1992) realizaram mais três estudos. No Estudo 1 consideraram 1.200 estudantes de Introdução à Psicologia de uma universidade estadunidense. Uma análise de componentes principais (rotação varimax) mostrou que os quatro fatores explicaram conjuntamente $60,7 \%$ da variância total, apresentando cargas fatoriais variando de 0,54 a 0,83. A consistência interna (alfa de Cronbach, a) desta medida, considerando o conjunto de 16 itens, foi 0,85 ; os coeficientes correspondentes para seus fatores específicos variaram de 0,73 (Filiação) a 0,80 (Pública).
O Estudo 2 considerou 83 estudantes do curso de Psicologia da mesma instituição, que responderam a medida de $A u-$ toestima Coletiva, a Escala de Rosenberg de Autoestima, a Escala de Sentimentos de Inadequação de Janis-Field, o Inventário de Autoestima de Coopersmith, a Escala de Desejabilidade Social de Marlowe-Crowne e a medida de Breckler, Greenwald e Wiggins (1986), que busca avaliar a orientação à tarefa e as estimas pública, privada e coletiva. 0 fator autoestima privada se correlacionou mais fortemente com a medida de Rosenberg $(r=0,43, p<0,001)$, a medida de Croopersmith $(r=0,48, p<0,001)$, a escala de inadequação $(r=0,41, p<$ $0,001)$ e a dimensão privada da medida de Breckler et al. (1986) $(r=0,42, p<0,001)$; e o fator filiação se correlacionou mais com a autoestima coletiva destes últimos autores ( $r=$ $0,44, p<0,001)$. Porém, mais importante, nenhum dos fatores da autoestima coletiva se correlacionou com a medida de desejabilidade social $(r=0,12, p>0,05)$. Nesse mesmo estudo foi realizada a aplicação da Escala de Autoestima Coletiva em duas ocasiões no intervalo de seis semanas, observando-se estabilidade temporal para a medida total $(r=0,68)$ e também para os fatores específicos: filiação $(r=0,58)$, pública $(r=$ $0,66)$, privada $(r=0,62)$ e identidade $(r=0,68)$. A consistência interna (alfa de Cronbach) dos fatores variou entre 0,73 (identidade) e 0,80 (privada).

Em seguida, considerando as amostras dos três estudos, Luhtanen e Crocker (1992) realizaram múltiplas análises fatoriais confirmatórias (estimador ML, Maximum Likelihood), testando quatro modelos: unifatorial, quatro fatores não correlacionados, quatro fatores correlacionados e hierárqui$\cos$ (quatro fatores de primeira ordem e um de segunda ordem). Consistentemente, os achados indicaram que o melhor modelo foi aquele com quatro fatores correlacionados, cujos valores médios de $X^{2} / \mathrm{gl}$ (razão qui-quadrado / graus de liberdade) e CFI (Comparative Fit Index) foram, respectivamente, 2,74 e 0,90 . Esta estrutura, contando com fatores correlacionados, encontra respaldo também na matriz de correlações interfatores, que em todos os estudos foram significativas ( $p$ $<0,05$; $r$ médio superior a 0,40). Portanto, corroborou-se a estrutura tetrafatorial incialmente observada; os alfas de Cronbach para os fatores específicos variaram de 0,71 (privada) a 0,86 (identidade).

Por fim, Utsey e Constatine (2006) realizaram um estudo para verificar a estrutura fatorial desta escala em uma amostra de negros estadunidenses, corroborando o modelo tetrafatorial originalmente proposto. Rossouw (2010) procurou conhecer a estrutura desta medida na África do Sul; apesar de ter encontrado índices de confiabilidades aceitáveis (variando entre 0,83 e 0,88 ), os indicadores de ajuste do modelo com quatro fatores não foram dentro do recomendado. Por outro lado, Bazińska (2015) buscou testar a mesma estrutura fatorial no contexto polonês, reunindo indicadores de ajuste 


\section{W'INTERACÃO EM PSICOLOGIA}

satisfatórios, apoiando a existência do modelo com quatro fatores correlacionados.

Em resumo, considerando que a EAC se mostra um instrumento psicometricamente promissor no contexto estrangeiro, carecendo, ainda, de evidências de validade e consistência interna no Brasil, teve-se como objetivo geral deste artigo descrever sua adaptação ao contexto brasileiro. Especificamente, realizaram-se dois estudos, objetivando: conhecer a estrutura fatorial e a consistência interna desta escala. No caso, testaram-se os modelos analisados por Luhtanen e Crocker (1992), i.e., unifatorial (todos os itens carregando em um fator geral de autoestima coletiva); tetrafatorial com fatores não correlacionados, fixando-se covariâncias iguais à zero; tetrafatorial com fatores correlacionados, isto é, permitindo que os parâmetros de covariâncias fossem estimados livremente; e, por fim, o modelo hierárquico, admitindo quatro fatores de primeira e um de segunda ordem (Estudo 1); e replicar os achados do estudo anterior, realizando análises fatoriais confirmatórias para comparar os quatro modelos, além de checar a consistência interna dos fatores da EAC (Estudo 2). Procurou-se, ainda, comprovar em que medida os alfas de Cronbach eram estáveis.

\section{ESTUD0 1. EVIDÊNCIAS PRELIMINARES DE VALIDA- DE FATORIAL E CONSISTENNCIA INTERNA}

Este estudo procurou conhecer a estrutura fatorial e consistência interna da EAC, tendo em conta uma amostra de conveniência de estudantes universitários. No caso, objetivouse testar os mesmos modelos analisados por Luhtanen e Crocker (1992), conforme anteriormente descritos.

\section{MÉTODO}

\section{Participantes}

Participaram deste estudo 497 estudantes de graduação de três áreas (ciências naturais, ciências humanas e ciências da saúde). A maioria deles foi do sexo masculino (51,5\%), solteira $(91,4 \%)$, católica $(48,4 \%)$ ou protestante $(22,6 \%)$, tendo idade média de 21 anos (variando de 16 a 56 anos). Eles se perceberam como moderadamente religiosos $[M=4,16$, $\mathrm{DP}=2,12$, em escala de oito pontos, variando de 0 (Nada religioso) a 7 (Totalmente religioso)] e de classe socioeconômica média (64,3\%). Tratou-se de amostra de conveniência, participando as pessoas que, presentes em sala de aula e concordando em colaborar com o estudo, o fizeram sem qualquer ganho ou ônus.

\section{Instrumentos}

Os participantes estiveram envolvidos em um projeto maior, que procurava conhecer os valores humanos e seus correlatos. Deste modo, eles responderam a EAC e perguntas sociodemográficas (idade, sexo, estado civil, religião, religiosidade e status socioeconômico) como parte do estudo. A versão original da EAC (Luhtanen \& Crocker, 1992) foi traduzida para o português por dois psicólogos bilíngues, sendo as traduções posteriormente comparadas para produzir uma versão preliminar, que foi logo submetida à validação semântica em um grupo com dez estudantes universitários. Os 16 itens desta escala são representados por quatro fatores de autoestima coletiva, como seguem: filiação (e.g., Sou um membro digno do meu país; Sinto que não tenho muito a oferecer ao meu país), privada (e.g., Geralmente me arrependo de fazer parte de meu país; No geral, sinto-me feliz por ser brasileiro), pública (e.g., No geral, o Brasil é considerado como bom por pessoas de outros países; Geralmente, os outros países respeitam o Brasil) e identidade (e.g., No geral, meu país tem muito pouco a ver com como me sinto sobre mim mesmo; Meu país é um reflexo importante de quem eu sou). Estes itens são respondidos em escala de 7-pontos, variando de 1 (Discordo totalmente) a 7 (Concordo totalmente).

\section{Procedimento}

Contataram-se incialmente as coordenações dos cursos, visando obter autorização para aplicação dos questionários. Após o consentimento, contatavam-se os professores das respectivas disciplinas e três bolsistas de Iniciação Científica (IC) do curso de Psicologia, devidamente treinados, ficaram responsáveis pela aplicação dos questionários. Estes foram preenchidos em contexto público de sala de aula, porém respondidos individualmente. Os participantes foram informados acerca do caráter voluntário de sua participação, garantido o anonimato e sigilo de suas respostas. Esclareceram-se as diretrizes éticas que regem as pesquisas com seres humanos, em consonância com a Resolução nº 512/16, do Conselho Nacional de Saúde, oportunidade em que os participantes foram previamente informados que poderiam deixar o estudo a qualquer momento sem penalização, embora se reiterasse a importância de sua colaboração. Os que concordaram em fazê-lo tiveram que assinar termo de consentimento livre e esclarecido, atestando sua ciência. Em média, 20 minutos foram suficientes para completar sua participação. 


\section{a merecicion PSICOLOGIA}

Valdiney Veloso Gouveia, Maria Gabriela Costa Ribeiro, Alessandro Teixeira Rezende, Thiago Medeiros Cavalcanti, Heloisa Bárbara Cunha Moizeís e Alex Sandro de Moura Grangeiro

\section{Análise de dados}

Os quatro modelos (unifatorial, quatro fatores não correlacionados, quatro fatores correlacionados e o modelo hierárquico) foram testados usando o programa Amos 20. Considerou-se como entrada a matriz de variância-covariância, usando o estimador ML (Maximum Likelihood). Os seguintes indicadores de ajuste foram considerados: a razão qui-quadrado / graus de liberdade (aceitando valores entre 2 e 3, admitindo aqueles abaixo de 5), GFI (Goodness of Fit Index), AGFI (Adjusted Goodness of Fit Index), NFI (Normed Fit Index), CFI (Comparative Fit Index) e RMSEA (Root Mean Square Error of Approximation). Valores para GFI, AGFI, NFI e CFI na casa de 0,90 ou superior e de 0,05 a 0,08 para o RMSEA (admitindo até 0,10 como limite superior) indicam um ajuste adequado do modelo (Hair, Black, Babin, Anderson, \& Tatham, 2015). Com o fim de comparar os modelos alternativos, utilizou-se a diferenças entre os qui-quadrados e os respectivos graus de liberdade $\left[\Delta X^{2}(\mathrm{gl})\right]$, penalizando o modelo com maior qui-quadrado.

\section{RESULTADOS}

Procurou-se inicialmente conhecer o melhor modelo fatorial para a EAC, testando os quatro modelos previamente descritos. Os resultados desta análise para as amostras de ambos os estudos (mais detalhes do Estudo 2 a seguir) podem ser vistos na Tabela 1 a seguir.

Conforme pode ser visto nesta tabela, apresentaram os piores indicadores de ajuste os modelos unifatorial (e.g., CFI $=0,72$ e RMSEA $=0,10$ ) e, principalmente, tetrafatorial, admi- tindo-se que seus fatores não estariam correlacionados (e.g., $\mathrm{CFI}=0,69$ e RMSEA $=0,10)$. O modelo com quatro fatores correlacionados apresentou indicadores de ajuste de meritórios a aceitáveis $(\mathrm{GFI}=0,90, \mathrm{CFI}=0,84$ e RMSEA $=0,08)$, sendo praticamente similares àqueles para o modelo hierárquico $(\mathrm{GFI}=0,90, \mathrm{CFI}=0,84$ e RMSEA $=0,08)$. Comparando os respectivos qui-quadrados e graus de liberdade, observa-se que tais modelos não foram estatisticamente diferentes $\left[\Delta X^{2}(2)\right.$ $=1,59, \mathrm{p}>0,05]$. Portanto, ambos são admissíveis, optandose, neste caso, pelo modelo mais parcimonioso, isto é, com quatro fatores correlacionados de primeira ordem, conforme se descreve na figura 1.

Cabe ressaltar que todas as saturações (lambdas, $\lambda$ ) foram estatisticamente diferentes de zero $(\lambda \neq 0 ; z>1,96, p<$ $0,05)$. Resta, entretanto, demonstrar a adequação da consistência interna dos quatro fatores, calculando-se seus respectivos alfa de Cronbach (a), tendo sido observados os seguintes coeficientes (Tabela 2$)$ : filiação $(a=0,58)$, pública $(a=0,82)$, privada $(a=0,53)$ e identidade $(a=0,63)$; o alfa para a escala total, isto é, reunindo os 16 itens, foi de 0,80 ; as correlações médias inter-itens variaram de 0,22 (pública) a 0,53 (privada). Todos os coeficientes de correlação entre fatores foram significativos $(p<0,001)$, variando de 0,16 (filiação e pública) a 0,51 (privada e identidade).

\section{DISCUSSÃO}

Em resumo, estas são as primeiras evidências de adequação psicométrica da EAC, tomando como grupo de referência o Brasil. Os achados foram na direção daqueles observados em estudos prévios sobre esta medida (Bazińska, 2015; Ros-

Tabela 1. Indicadores de Ajuste dos Quatro Modelos de Autoestima Coletiva

\begin{tabular}{|c|c|c|c|c|c|c|c|}
\hline & Modelo & $\chi^{2}(\mathrm{gl})$ & GFI & AGFI & $\mathrm{NFI}$ & $\mathrm{CFI}$ & RMSEA (IC95\%) \\
\hline \multirow{4}{*}{ 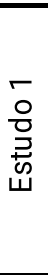 } & Um fator & $595,35(104)$ & 0,86 & 0,82 & 0,68 & 0,72 & $\begin{array}{c}0,098 \\
(0,090-0,105)\end{array}$ \\
\hline & Quatro fatores ortogonais & $648,31(104)$ & 0,84 & 0,80 & 0,66 & 0,69 & $\begin{array}{c}0,103 \\
(0,095-0,110)\end{array}$ \\
\hline & Quatro fatores oblíquos & $378,26(98)$ & 0,90 & 0,86 & 0,80 & 0,84 & $\begin{array}{c}0,076 \\
(0,068-0,084)\end{array}$ \\
\hline & Hierárquico & $379,85(100)$ & 0,90 & 0,86 & 0,80 & 0,84 & $\begin{array}{c}0,075 \\
(0,067-0,083)\end{array}$ \\
\hline \multirow{4}{*}{ 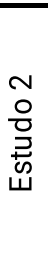 } & Um fator & $528,22(104)$ & 0,85 & 0,80 & 0,73 & 0,77 & $\begin{array}{c}0,102 \\
(0,094-0,111)\end{array}$ \\
\hline & Quatrofatores ortogonais & $765,08(104)$ & 0,79 & 0,72 & 0,61 & 0,64 & $\begin{array}{c}0,128 \\
(0,119-0,136)\end{array}$ \\
\hline & Quatro fatores oblíquos & $362,09(98)$ & 0,89 & 0,84 & 0,82 & 0,86 & $\begin{array}{c}0,083 \\
(0,074-0,092)\end{array}$ \\
\hline & Hierárquico & $364,69(100)$ & 0,89 & 0,85 & 0,81 & 0,86 & $\begin{array}{c}0,082 \\
(0,073-0,092)\end{array}$ \\
\hline
\end{tabular}

Nota: Todos os qui-quadrados tiveram $p<0,001$. Ns (números de participantes) $=497$ e 391 nos estudos 1 e 2 , respectivamente. 
Valdiney Veloso Gouveia, Maria Gabriela Costa Ribeiro, Alessandro Teixeira Rezende, Thiago Medeiros Cavalcanti, Heloisa Bárbara Cunha Moizeís e Alex Sandro de Moura Grangeiro

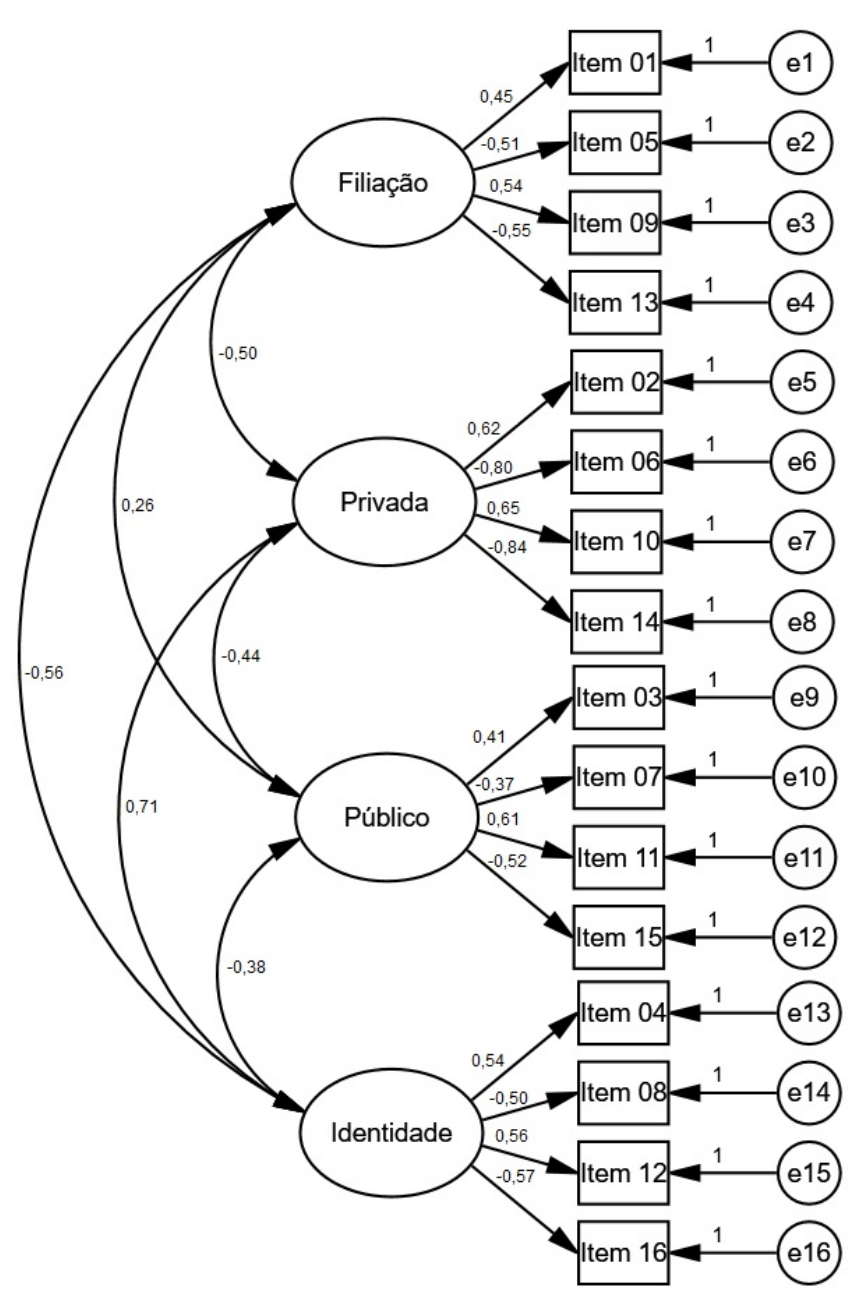

Figura 1.Modelo de quatro fatores correlacionados. souw, 2010; Utsey \& Constatine, 2006), incluindo o estudo original de sua elaboração (Luhtanen \& Crocker, 1992). Não obstante, parece necessário checar em que medida esses achados são replicados em uma amostra independente, considerando pessoas da população geral, o que motivou um segundo estudo.

\section{ESTUDO 2. COMPROVAÇÃO DA ESTRUTURA FATO- RIAL E CONSISTENNCIA INTERNA}

Este estudo, como ficou evidenciado anteriormente, focou em replicar os achados prévios, realizando análises fatoriais confirmatórias para comparar os quatro modelos, além de checar a consistência interna dos fatores da EAC. Procurou-se, ainda, comprovar em que medida seus alfas de Cronbach eram estáveis ou flutuavam para além de erros aleatórios.

\section{MÉTODO}

\section{Participantes}

Participaram deste estudo 391 pessoas da população geral, os quais apresentaram idade média de 23,3 (variando de 18 a 57 anos), predominantemente do sexo masculino $(60,9 \%)$, solteira $(85,7 \%)$, católica $(39,6 \%)$ ou protestante $(18,2 \%)$, autodeclarando-se medianamente religiosos $[M=$ $3,79, D P=1,99$; escala de oito pontos, variando de $(0=\mathrm{Nada}$ religioso) a 7 (Totalmente religioso)]. Tratou-se de amostra de conveniência, participando pessoas que foram convidadas na internet, aceitando a colaborar voluntariamente do estudo.

Tabela 02: Estatísticas descritivas, consistência interna e correlações da Escala de Autoestima Coletiva

\begin{tabular}{|c|c|c|c|c|c|}
\hline \multirow{2}{*}{ Estatísticas } & \multicolumn{4}{|c|}{ Escala } & \multirow{2}{*}{ Total } \\
\hline & Filiação & Privada & Pública & Identidade & \\
\hline \multicolumn{6}{|l|}{ Escala M (DP) } \\
\hline Estudo 1 & $21,46(3,89)$ & $19,17(5,28)$ & $15,49(4,02)$ & $15,95(4,68)$ & $72,07(12,70)$ \\
\hline Estudo 2 & $21,91(4,22)$ & $19,41(5,98)$ & $15,14(4,41)$ & $15,83(5,11)$ & $72,28(14,86)$ \\
\hline \multicolumn{6}{|l|}{$r_{\text {Média }}$ (inter-item) } \\
\hline Estudo 1 & 0,26 & 0,53 & 0,22 & 0,30 & 0,40 \\
\hline Estudo 2 & 0,30 & 0,59 & 0,27 & 0,29 & 0,24 \\
\hline \multicolumn{6}{|l|}{$r_{\text {Média }}$ item-total) } \\
\hline Estudo 1 & 0,37 & 0,64 & 0,32 & 0,41 & 0,40 \\
\hline Estudo2 & 0,41 & 0,69 & 0,37 & 0,39 & 0,43 \\
\hline \multicolumn{6}{|l|}{ Alfa de Cronbach } \\
\hline Estudo 1 & 0,58 & 0,82 & 0,53 & 0,63 & 0,80 \\
\hline Estudo 2 & 0,63 & 0,85 & 0,59 & 0,62 & 0,84 \\
\hline \multicolumn{6}{|l|}{ Correlações } \\
\hline Filiação & & $0,36(0,40)$ & $0,16(0,26)$ & $0,34(0,41)$ & \\
\hline Privada & & & $0,33(0,42)$ & $0,51(0,61)$ & \\
\hline Pública & & & & $0,22(0,30)$ & \\
\hline
\end{tabular}

Nota: Cada escala é composta por quatro itens. N (número de participantes) = 497 e 391 nos Estudos 1 e 2, respectivamente. Todas as correlacoes foram estatisticamente significativas $(p<0,001)$. Os coeficientes entre parênteses correspondem ao Estudo 2. 


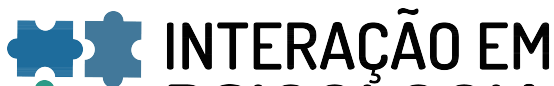 PSICOLOGIA}

Valdiney Veloso Gouveia, Maria Gabriela Costa Ribeiro, Alessandro Teixeira Rezende, Thiago Medeiros Cavalcanti, Heloísa Bárbara Cunha Moizeís e Alex Sandro de Moura Grangeiro

\section{Instrumentos}

Os participantes responderam os mesmos instrumentos do Estudo 1, fazendo igualmente parte de um projeto maior que procurou conhecer os valores e seus correlatos na população geral, isto é, não universitária. Portanto, todos responderam a EAC e perguntas sociodemográficas, que foram detalhadas no Estudo 1.

\section{Procedimento}

Os instrumentos foram organizados na plataforma virtual LimeSurvey e disponibilizados em homepage com acesso público (http://vvgouveia.net). 0 link da pesquisa foi copiado e compartilhado pelos autores nas redes sociais (e.g., Facebook, Instagram), além de ser postado em grupos públicos e encaminhado para endereços de e-mails de pessoas que participaram de estudos prévios. Em média, 15 a 20 minutos foram suficientes para concluir a participação no estudo, devendo os participantes concordarem com o termo de consentimento livre e esclarecido, em conformidade com o que determina a Resolução $n^{\circ} 512 / 16$, do Conselho Nacional de Saúde.

\section{Análises de dados}

Utilizou-se o mesmo programa estatístico do estudo anterior (Amos, versão 20), realizando as análises fatoriais confirmatórias que tiveram como entrada a matriz de variânciacovariância, admitindo-se o estimador ML. Adotaram-se os mesmos pontos de corte para os indicadores de ajuste do modelo. Além dessas análises, decidiu-se, ainda, comparar os alfas de Cronbach deste estudo com aqueles do Estudo 1,

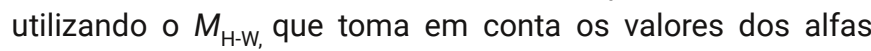
ponderando o número de itens de cada fator e os tamanhos das amostras utilizadas (Hakstian \& Whalen, 1976).

\section{RESULTADOS}

Testaram-se quatro modelos fatoriais. Conforme é possível observar na Tabela 1, o pior modelo foi aquele com quatro fatores ortogonais (e.g., $\mathrm{CFI}=0,64$ e RMSEA $=0,13$ ), seguido do que admitiu um único fator geral de autoestima coletiva (e.g., $\mathrm{CFI}=0,77$ e RMSEA = 0,10). Uma vez mais, os modelos com quatro fatores oblíquos (e.g., $\mathrm{CFI}=0,86$ e RMSEA $=0,08$ ) e aquele hierárquico (e.g., $\mathrm{CFI}=0,86$ e RMSEA $=0,08$ ), admitindo esses fatores e um de segunda ordem, foram os mais promissores. Comparando estes dois modelos, observou-se que não foram estatisticamente diferentes $\left[\Delta X^{2}(2)=2,60, p>\right.$ $0,05]$. Deste modo, endossa-se o modelo com quatro fatores oblíquos, que é o mais parcimonioso. Acentua-se que as saturações de seus itens nos respectivos fatores foram todas diferentes de zero $(\lambda \neq 0 ; z>1,96, p<0,05)$.

Quanto ao parâmetro de consistência interna desta medida, na Tabela 2 é possível observar os resultados correspondentes. No caso, checaram-se os alfas de Cronbach para o conjunto de seus 16 itens $(a=0,84)$, bem como para os fatores específicos, como seguem: filiação $(a=0,63)$, pública $(a=$ $0,85)$, privada $(a=0,59)$ e identidade $(a=0,62)$. As correlações médias inter-itens variaram de 0,27 (pública) a 0,59 (privada). Observou-se, ainda, que as correlações entre fatores foram significativas $(p<0,001)$, variando de 0,26 (filiação e pública) a 0,61 (privada e identidade). Por fim, quando estes coeficientes de consistência interna foram comparados com aqueles do Estudo 1, comprovou-se que nenhum se mostrou estatisticamente diferente $\left[0,07\right.$ (identidade) $<M_{\mathrm{H}-\mathrm{W}}<3,42$ (privada), $p>0,05]$.

\section{DISCUSSÃO}

Conforme se depreende nos resultados anteriormente apresentados, corrobora-se a adequação de pensar a EAC como sendo tetrafatorial, consoante com o que propuseram seus autores (Luhtanen \& Crocker, 1992). Estes achados e os do estudo anterior serão detalhados a seguir.

\section{DISCUSSÃO GERAL}

Considerando os objetivos iniciais deste artigo, isto é, adaptar a EAC, reunindo evidências de sua validade fatorial e consistência interna, estima-se que tenham sido alcançados. Concretamente, realizaram-se dois estudos com amostras adequadas para as análises estatísticas realizadas (Tabachnick \& Fidell, 2013), que mostraram resultados que dão suporte para a estrutura com quatro fatores correlacionados, os quais apresentaram indicadores de consistência interna (alfa de Cronbach e homogeneidade) que estão em linha com o que se esperaria, ainda que um pouco abaixo daqueles relatados para estudos em outros países (Bazińska, 2015; Rossouw, 2010; Utsey \& Constatine, 2006). Entretanto, cabem algumas considerações específicas.

Quanto às evidências de validade fatorial, diferentemente do estudo de Luhtanen e Crocker (1992) em que a estrutura com quatro fatores oblíquos foi claramente a melhor, nos dois estudos anteriormente relatados esta estrutura não se diferenciou daquela hierárquica. Esses achados foram similares àqueles observados na África do Sul em que não houve diferença entre os modelos hierárquico e tetrafatorial (quatro 


\section{H. INTERACÃO EM PSICOLOGIA}

Valdiney Veloso Gouveia, Maria Gabriela Costa Ribeiro, Alessandro Teixeira Rezende, Thiago Medeiros Cavalcanti, Heloisa Bárbara Cunha Moizeís e Alex Sandro de Moura Grangeiro fatores correlacionados). Na ocasião a autora sugeriu que a multiculturalidade daquele país pode ter tido papel preponderante, sugerindo a necessidade de definir na população-meta o conceito de autoestima coletiva (Rossouw, 2010). Portanto, parece razoável admitir os quatro fatores de primeira ordem, mas também um geral de segunda ordem, que reúne aqueles, talvez fruto de um contexto que evidencia uma pluralidade cultural, como ocorre no estudo de Rassouw (2010), podendose de certo modo diferenciar do contexto estadunidense em que essa medida foi elaborada.

No que diz respeito à consistência interna dessa escala, parece claro que os resultados de Luhtanen e Crocker (1992) foram mais promissores, incluindo aqueles observados na África do Sul (Rossouw, 2010). Contudo, é importante ressaltar que, embora os coeficientes das dimensões de autoestima nomeadas como filiação e pública tenham sido abaixo do ponto de corte comumente recomendado na literatura $(0,70$; Gouveia, Santos, \& Milfont, 2009), há que se ponderar o número reduzido de itens por fator, o que pode comprometer este parâmetro. A propósito, um indicador menos influenciado é a homogeneidade do fator, correspondendo à correlação média inter-itens, que se recomenda que seja igual ou superior a 0,20 (Clark \& Watson, 1995). É importante observar, ainda, que os alfas de Cronbach foram estáveis, não mudando substancialmente em razão da amostra (Tabachnick \& Fidell, 2013).

Apesar desses achados promissores, que apresentam uma medida que poderá ser utilizada para fins de pesquisa, não é possível deixar de reconhecer limitações potenciais deste estudo. Talvez em razão da diversidade cultural do Brasil, reunindo pessoas e estilos de vida tão diferentes nas regiões e mesmo estados, tomar esta nação como um grupo pode não ser o mais adequado, o que tem potencial para se refletir na variabilidade de resposta intra-sujeito, que repercute na menor homogeneidade da medida. Contudo, esta não é uma característica do instrumento em si, que poderá ser adaptado para outros grupos sociais. Porém, precisar-se-á ter em conta que grupo considerar, correndo o risco de contar com uma medida mais flutuante.

Em termos de estudos futuros, ressalta-se a importância de investir na análise de parâmetros complementares de validade e precisão desta medida. Por exemplo, valerá a pena, como o fizeram Luhtanen e Crocker (1992), checar a validade discriminante desta medida com relação à desejabilidade social. Como um país coletivista, o Brasil é potencialmente mais suscetível de apresentar desejabilidade social, o que pode influenciar nas pontuações de autoestima coletiva (Soares et al., 2016). No caso do parâmetro de precisão, valerá a pena conhecer algo mais sobre a estabilidade temporal desta medida; o estudo de Luhtanen e Crocker (1992) oferece alguma evidência, porém tais achados ainda são escassos, cabendo considerar uma amostra mais substancial e maior tempo entre as aplicações (esses autores consideraram seis semanas).

Por fim, estudos sobre a autoestima coletiva parecem fundamentais para entender muitos dos construtos culturais (Ferreira, Fischer, Porto, Pilati, \& Milfont, 2012; Miura, 2012). Por exemplo, o "jeitinho brasileiro", que pode ser compreendido como a criação de estratégias para resolução de problemas em que a pessoa utiliza da influência social combinada a estratégias perspicazes para alcançar objetivos, mesmo que quebre algumas regras formais (Ferreira, et al., 2012). Em outras palavras, o jeitinho brasileiro é tido como um traço típico do povo deste país e carece de maior compreensão; afinal, assumir este perfil pode implicar maior ou menor autoestima coletiva. Porém, outros construtos poderão igualmente se beneficiar com uma medida de autoestima coletiva, contribuindo para entender se o julgamento que o indivíduo realiza em relação ao grupo social de pertença ocorre a partir da sua socialização, isto é, passagem de uma a outra geração, como também se verifica em outros fenômenos, a exemplo dos valores humanos (Gouveia, 2016; Gouveia, Vione, Milfont \& Santos, 2015). É possível que onde a autoestima coletiva seja mais forte, ou seja, a avaliação positiva dentro do grupo social, as pessoas costumem aderir ou repassar mais os valores prevalentes na cultura. Além do mais, seria importante comparar como diferem os grupos sociais no Brasil, devido sua constituição plural e étnica. Portanto, abre-se com estes estudos uma avenida de possibilidades no contexto brasileiro, não apenas de estudos psicométricos, mas, sobretudo, aqueles que permitam conhecer os antecedentes e consequentes da autoestima coletiva.

\section{DECLARAÇÃO DE FINANCIAMENTO}

A pesquisa relatada no manuscrito foi financiada parcialmente pela bolsa de produtividade em pesquisa do CNPQ nível $1 \mathrm{~A}$ do primeiro autor.

\section{DECLARAÇÃO DA CONTRIBUIÇÃO DOS AUTORES}

V. V. G. responsável pela redação inicial e conceitualização; M. G. C. R. e A. T. R. realizaram a investigação e coleta de dados; T. M. C., H. B. C. M. e A. S. M. G. fizeram as análises e edição.

\section{REFERÊNCIAS}

Andersen, S. M., \& Cole, S. W. (1990). “Do I know you?”: The role of significant others in general social perception. Journal of Personality and Social Psychology, 59, 384-399. http://dx.doi.org/10.1037/0022-3514.59.3.384 


\section{W INERACÄOEM PSICOLOGIA}

Bazińska, R. (2015). Validation of the Polish version of the collective self-esteem scale. Current Issues in Personality Psychology, 3, 125-137. http://dx.doi.org/10.5114/cipp. 2015.52891

Breckler, S. J., Greenwald, A. G., \& Wiggins, E. C. (1986). Public, private, and collective self-evaluation: Measurement of individual differences. Paper presented at the International Research and Exchange Board Conference on Self and Social Involvement, Princeton, NJ.

Clark, L. A., \& Watson, D. (1995). Constructing validity: Basic issues in objective scale development. Psychological Assessment, 7, 309 - 321. http://dx.doi.org/10.1037/10403590.7.3.309

Crocker, J., Luhtanen, R., Blaine, B., \& Broadnax, S. (1994). Collective self-esteem and psychology well-being among white, black, and Asian college students. Society for Personality and Social Psychology, 20, 503-513. http:// dx.doi.org/10.1177/0146167294205007

Diener, E., \& Diener, M. (2009). Cross-cultural correlates of life satisfaction and self-esteem. In: E. Diener (Ed.), Culture and well-being: The collected works of Ed Diener (pp.7191). Netherlands: Springer.

Du, H., King, R. B., \& Chi, P. (2012). The development and validation of the Relational Self-Esteem Scale. Personality and Social Psychology, 53, 258-264. http://dx.doi.org/10.1111/ j.1467-9450.2012.00946.x

Ferreira, M. C., Fischer, R., Porto, J. B., Pilati, R., \& Milfont, T. L. (2012). Unraveling the mystery of Brazilian Jeitinho: A cultural exploration of social norms. Personality and Social Psychology Bulletin, 38, 331-344. http://dx.doi.org/ 10.1177/0146167211427148

Gouveia, V. V. (2016). Introdução à teoria funcionalista dos valores. Em V. V. Gouveia (Ed.), Teoria funcionalista dos valores humanos: Áreas de estudo e aplicações (pp. 13-27). São Paulo: Vetor.

Gouveia, V. V., Santos, W. S., \& Milfont, T. L. (2009). O uso da estatística na avaliação psicológica: comentários e considerações práticas. Em C. S. Hutz. (Ed.), Avanços e Polêmicas em Avaliação Psicológica: Em Homenagem a Jurema Alcides Cunha (pp. 127-155). São Paulo: Casa do Psicólogo.

Gouveia, V. V., Vione, K. C., Milfont, T. L., \& Santos, W. S. (2015). Guiding actions and expressing needs: On the psychological functions of values. Psykhe, 24, 1-14. http:// dx.doi:10.7764/psykhe.24.2.884

Hair, J. F. J., Black, W. C., Babin, B. J., \& Anderson, R. E. (2015). Multivariate Data Analysis (7a Ed.). Upper Saddle River, NJ: Prentice Hall.

Hakstian, A. R., \& Whalen, T. E. (1976). A k-sample significance test for independent alpha coefficients. Psychometrika, 41, 219-231. http://dx.doi.org/10.1007/BF02291840
Hassan, G., Rousseau, C., \& Moreau, N. (2013). Ethnic and religious discrimination: The multifaceted role of religiosity and collective self-esteem. Transcultural Psychiatry, 50, 475-492. http://dx.doi.org/10.1177/1363461513495586

Hutz, C. S., \& Zanon, C. (2011). Revisão da adaptação, validação e normatização da Escala de Autoestima de Rosenberg. Avaliação Psicológica, 10, 41-49.

Kernis, H. (2005). Measuring self-esteem in context: The importance of stability of selfesteem in psychological functioning. Journal of Personality, 73, 1569-1605. http:// doi: $10.1111 /$ j.1467-6494.2005.00359.x

Luhtanen, R., \& Crocker, J. (1992). A Collective Self-Esteem Scale: Self-evaluation of one's social identity. Personality and Social Psychology Bulletin, 18, 302-318. http:// dx.doi.org/10.1177/0146167292183006

Melo, C. V. G. (2014). Estratégias de enfrentamento de pessoas negras e com deficiência frente ao duplo estigma. (Dissertação de Mestrado não publicada). Departamento de Psicologia, Universidade Federal da Paraíba, João Pessoa, PB.

Miura, M. A. (2012). Entre a simpatia e a corrupção: Análise das dimensões constituintes do Jeitinho Brasileiro. (Dissertação de Mestrado não publicada). Departamento de psicologia, Universidade de Brasília, Brasília, DF.

Paiva, M. A. C., \& Romero, E. (2004). Identidade social e autoestima em participantes de grupos de dança flamenca: $A$ contribuição pedagógica e psicológica ao ser do homem em movimento. Fitness \& Performance Journal, 3, 28-32.

Rossouw, A. (2010). Confirmatory factor analysis of the collective self-esteem scale. (Dissertação de Mestrado não publicada), Psychology department, University of Pretoria, Pretoria, África do Sul.

Simsek, O. F. (2013). Structural relations of personal and collective self-esteem to subjective well-being: Attachment as moderator. Social Indicators Research, 110, 219-236. http://dx.doi.org/10.1007/s11205-011-9927-1

Soares, A. K. S., Lopes, G. S., Rezende, A. T., Ribeiro, M. G. C., Santos, W. S., \& Gouveia, V. V. (2016). Escala de Desejabilidade Social Infantil (EDSI): Evidência de validade fatorial e consistência interna. Avances en Psicología Latinoamericana, 34, 383-394. http://dx.doi.org/10.12804/ apl34.2.2016.11

Souza, D. B. L., \& Ferreira, M. C. (2005). Auto-estima pessoal e coletiva em mães e não-mães. Psicologia em Estudo, 10, 19-25.

Sowislo, J. F., \& Orth, U. (2013). Does low self-esteem predict depression and anxiety? A meta-analysis of longitudinal studies. Psychological Bulletin, 139, 213-240. http:// dx.doi.org/10.1037/a0028931 


\section{N"INTERACÃO EM \\ EF PSICOLOGIA}

Valdiney Veloso Gouveia, Maria Gabriela Costa Ribeiro, Alessandro Teixeira Rezende, Thiago Medeiros Cavalcanti, Heloisa Bárbara Cunha Moizeís e Alex Sandro de Moura Grangeiro

Suit, D. A. V. (2012). Trajetórias de mulheres que vivem com o HIV: Um estudo a respeito de ideologia de gênero, autoestima e bem-estar subjetivo. (Dissertação de Mestrado não publicada). Departamento de Psicologia, Universidade Federal da Bahia, Salvador, BA.

Tabachnick, B. G., \& Fidell, L. S. (2013). Using Multivariate Statistics ( $6^{\mathrm{a}} \mathrm{ed}$.). Nova lorque: Allyn \& Bacon.

Tajfel, H. (1981). Human groups and social categories: Studies in social psychology. Cambridge: Cambridge University Press.

Utsey, S. O., \& Constantine, M. G. (2006). A confirmatory test of the underlying factor structure of scores on the collective self-esteem scale in two independent samples of black Americans. Journal of Personality Assessment, 86, 172179. http://dx.doi.org/10.1207/s15327752jpa8602_06

Recebido em: 19/07/2017

Primeira decisão editorial em: 16/10/2017

Aceito em: 05/01/2018 